\title{
Editorial
}

\section{Pestiviruses: old enemies and new challenges}

\author{
Received 30 March 2015; Accepted 13 April 2015
}

\begin{abstract}
The genesis for this special issue on pestiviruses was a joint meeting on pestiviruses organized by the US BVDV Symposia Committee and the European Society for Veterinary Virology that was held October 14 and 15, 2014 in Kansas City, MO. The theme of the meeting was "Pestiviruses: Old enemies and new challenges". The impetus for this joint effort was the recognition that regional approaches to disease control are at odds with the worldwide traffic in animal products and biologics. Further, the control of newly recognized pestiviruses, such as HoBi-like viruses, is more effective when approached as a global challenge rather than any one nation's problem. The joint meeting featured talks by researchers from North America, South America, Australia and Europe. The papers in this issue arose from keynote talks presented at the joint meeting and are organized around the following themes; pestiviruses and the immune system, genetic variability, the emergence of new pestiviruses and pestivirus control programs.
\end{abstract}

Keywords: pestiviruses, eradication, emerging, impact, US BVDV Symposium, ESVV Pestivirus Meeting.

\section{Introduction}

The genesis for this special issue on pestiviruses was a joint meeting on pestiviruses organized by the US BVDV Symposia Committee and the European Society for Veterinary Virology that was held 14 and 15 October 2014. The theme of the meeting was 'Pestiviruses: Old enemies and new challenges'. Over the past two decades a consortium of researchers, diagnosticians, government regulators, veterinary consultants, and field veterinarians in the USA have organized five symposia centered on bovine viral diarrhea virus (BVDV). The purpose of these symposia was to provide a platform for the report of both cutting edge basic research and the application of that research to practical control practices for bovine viral diarrhea virus. Similarly the European Society of Veterinary Virology, beginning in 1990, has held a series of eight international meetings on pestiviruses. These meetings provided an opportunity for presentation of research findings and discussion of pestivirus control strategies, including not just BVDV but also classical swine fever virus (CSFV), border disease virus (BDV) and emerging pestivirus species. The October 2014 meeting was the first time that the two groups collaborated in the organization of a joint symposium.

*Corresponding author. E-mail: julia.ridpath@ars.usda.gov
The impetus for this joint effort was the recognition that regional approaches to disease control are at odds with the worldwide traffic in animal products and biologics. Further, the control of newly recognized pestiviruses, such as HoBi-like viruses, is more effective when approached as a global challenge rather than any one nation's problem.

The joint meeting featured talks by researchers from North America, South America, Australia and Europe. The papers in this issue arose from keynote talks presented at the joint meeting and are organized around the following themes: pestiviruses and the immune system, genetic variability, the emergence of new pestiviruses and pestivirus control programs.

\section{Pestiviruses and the immune system}

Clinical presentations following acute infection with pestiviruses vary from severe, with high death losses, to mild with few clinical signs, depending on pestivirus species, strain virulence, and host. One constant among pestivirus infection is that a primary site of replication is immune tissue. Pestivirus replication in immune tissues results in altered cell function in some lymphoid populations and cell death in others. The resulting immune suppression occurs in all acute pestivirus infections regardless of the 
severity of clinical presentation or host. In addition, pestiviruses possess the ability to cross the placenta and infect the fetus. The outcome of fetal infection, which varies by stage of gestation and pestivirus species, includes fetal death, congenital defects, and life-long persistent infections. Persistent infections are maintained due to establishment of immunotolerance in the fetus. The ability to induce life-long immunotolerance appears to be unique to pestiviruses.

The first three papers in this issue address different aspects of the impact of pestivirus infection on immune tissue. The first paper entitled 'Immune response to bovine viral diarrhea virus-looking at newly defined targets' by Chase et al., reviews the published literature that addresses depletion of lymphocytes, dysregulation of neutrophil and natural killer cell function and the roles of receptors and cytokines in BVDV acute infections. In addition, new information on the effect of BVDV on immune development in the fetal liver and the role of resident macrophages is discussed. The second paper, 'Innate and adaptive immune responses to in utero infection with bovine viral diarrhea virus', by Hansen et al., summarizes a series of experiments that examined the impact of BVDV fetal infection on interferon (IFN)-associated pathways. The hypothesis that the maintenance of persistent infections is associated with impaired induction of IFN- $\gamma$ is discussed. The third paper 'BVDV vaccination in North America: risks versus benefits' by Griebel looks at a different aspect of immune suppression. In this paper, the available literature that examines immune suppression due to vaccination is reviewed and the benefit to risk ratio for vaccination is discussed.

\section{Pestivirus genetic variability}

The pestiviruses, similar to other viruses with single-stranded RNA genomes, have a high rate of genomic variability. The high rate of variability leads to genomic drift, which can give rise to regional differences in viral genotypes and subgenotypes, variation in virulence, and antigenic differences. This inherent heterogeneity works in the virus's favor by providing a large number of variant genomes available for natural selection. The degree of variation between pestivirus genotypes can result in vaccine failure. Ideally, vaccines should stimulate complete immunity, providing $100 \%$ protection against disease, viremia, shedding, and $100 \%$ fetal protection in vaccinates when challenged with a range of diverse viruses. Genomic variability that results in antigenic variability among circulating pestivirus in a region could reduce the level of protection afforded by vaccines. Improving vaccine protection may require first determining which genotypes and subgenotypes of a pestivirus are in circulation in a region and then designing a vaccine that contains antigens from those genotypes and subgenotypes.

The two papers making up this section address the impact of genomic variability on the regional distribution of genotypes, virulence, and control. The paper 'Genetic variability and distribution of Classical swine fever virus' by Beer et al., reviews the current literature regarding geographical patterns of classical swine fever virus (CSFV) diversity and possible genetic virulence markers. The paper 'Impact of species and subgenotypes of BVDV on control by vaccination' by Fulton is a review of published acute challenge and fetal protection studies done in US cattle to evaluate vaccine efficacy. The subgenotypes in BVDV vaccines and challenge strains are listed and publications focusing on the duration of immunity afforded by vaccines and the issue of contamination of summarized.

\section{Emergence of new pestiviruses}

The term "emerging viruses" includes novel viruses that suddenly appear in a host species without any historical precedence, viruses that have jumped from one host species to another and viruses that have undergone a change in virulence. While pestiviruses were first isolated from domestic hosts in Europe, the $\mathrm{UK}$, and the USA, it is now apparent that pestiviruses are endemic in domestic and wildlife species worldwide. Originally, the members of this genus were defined by the domestic host from which they were isolated, so that pestiviruses isolated from swine were called hog cholera virus (later CSFV), those from cattle were called BVDV and those from small ruminants were called BDV. Phylogenetic analysis revealed that there were two species of virus within the BVDV group, now referred to as BVDV1 and BVDV2. The International Committee on Taxonomy of Viruses (ICTV) recognizes CSFV, BVDV1, BVDV2, and BDV, as the four species that make up the pestivirus genus (Simmonds et al., 2014). However, the ICTV report also notes that there are four putative pestivirus species, pronghorn virus, giraffe virus, Bungowannah virus, and HoBi-like virus (also known as atypical bovine pestivirus or BVDV3). In addition, new clinical presentations resulting from infection with recognized species as well as infection of new hosts have been reported. The different categories of emerging pestiviruses are reflected in the four papers included in this section. The paper 'Emerging pestiviruses infecting domestic and wildlife hosts', by Ridpath, reviews the categories of emerging viruses and discusses examples of each within the pestivirus genus. The following three papers take a more in-depth look at specific 'emerging' pestiviruses. The papers 'Bungowannah virus - a probable new species of pestivirus - what have we found in the last 10 years?', by Kirkland et al., and "HoBi-like viruses - the typical 'atypical bovine pestivirus", by Bauermann and Ridpath, review the detection, characterization and impact on agriculture of two of the putative pestivirus species. The paper 'The two sides of border disease in Pyrenean chamois (Rupicapra pyrenaica): silent persistence and population collapse', by Marco et al., reviews an outbreak of disease associated with a host jump and change in virulence.

\section{Pestivirus control programs}

Regional eradication programs have been instituted for both CSFV and BVDV with varying levels of success. The paper 
'Perspective on BVDV control programs', by Givens and Newcomer, reviews the issues facing control programs for BVDV in the USA and Canada. Of particular interest in this paper is a discussion of the dynamics involved in decisionmaking by US cattle producers. The authors identify hurdles to BVDV control and eradication in North America. The paper 'Pestivirus control programs - how far have we come and where are we going?', by Moennig and Beecher, reviews control programs from a more international viewpoint. The authors analyze control programs that have been effective in reducing/eradicating different pestivirus species and outline the program components necessary for successful eradication.

[Note: Mention of trade name, proprietary product, or specified equipment does not constitute a guarantee or warranty by the USDA and does not imply approval to the exclusion of other products that may be suitable. USDA is an Equal Opportunity Employer.]

Julia F. Ridpath* and John D. Neill Ruminant Diseases and Immunology Research Unit, National Animal Disease Center, ARS/USDA, Ames, LA, USA

\section{Acknowledgments}

This symposium was made possible by a grant from the National Institute of Food and Agriculture of the USDA (NIFA) and sponsorship from the following companies: Merck Animal Health, Idexx Laboratories, Boehringer Ingelheim Vetmedica, Zoetis Animal Health, Qiagen, Novartis Animal Health, Merial and Elanco. Mention of trade name, proprietary product, or specified equipment does not constitute a guarantee or warranty by the USDA and does not imply approval to the exclusion of other products that may be suitable. USDA is an Equal Opportunity Employer.

\section{Reference}

Simmonds P, Becher P, Collett MS, Gould EA, Heinz FX, Meyers G, Monath T, Pletnev A, Rice CM, Stiasny K, Thiel H-J, Weiner A and Bukh J (2014). Family Flaviviridae. In: MQ King Andrew, Lefkowitz Elliot (eds) Classification and Nomenclature of Viruses Ninth Report of the International Committee on Taxonomy of Viruses. Waltham, MA, USA: Elsevier Academic Press, pp. 1000-1020. 